\title{
Air quality street level simulations by using an integrated mesoscale air quality modelling system (MM5-CMAQ-EMIMO) and a CFD model (MIMO): Madrid case study
}

\author{
R. San José ${ }^{1}$, J. L. Pérez ${ }^{1}$ \& R. M. González ${ }^{2}$ \\ ${ }^{1}$ Environmental Software and Modelling Group, \\ Computer Science School, Technical University of Madrid, Spain \\ ${ }^{2}$ Department of Meteorology and Geophysics, Faculty of Physics, \\ Complutense University of Madrid; Spain
}

\begin{abstract}
The advances in the capability of Computational Fluid Dynamics models and Air Quality Modelling Systems during the last decade have been quite substantial. The increase in computer capabilities and in the knowledge of turbulence parameterization and numerical schemes has also been very significant during the last ten years. On the other hand, there is a considerable public interest in information relating to the "real" pollution they are exposed to when they are walking in the street, going to work or even during the period they are driving a car from/to work or other daily activities. At street level the differences in the concentration values at both sides of a street can be important, particularly, for instance, in relation to photochemical production during the summer time in Mediterranean regions. In this contribution we have used the CFD model MIMO (U. of Karlsruhe (Germany)) and the mesoscale air quality modelling system MM5-CMAQ-EMIMO (NCEP / EPA / Technical University of Madrid) to simulate the impact of different emission reduction scenarios in the downtown area of Madrid City. These complex systems could evaluate the impact of several urban strategic emission reduction measures such as reduction of private traffic, increase of public transportation, impact on the introduction of new fuel cell vehicles, etc. Also, they could be used for the analysis of pollution concentrations at different heights (buildings) and in different areas of urban neighbourhoods.
\end{abstract}




\section{Introduction}

Air dispersion in urban areas is affected by atmospheric flow changes produced by building street geometry and aerodynamic effects. The traffic flow, emissions and meteorology are also play an important role. Microscale air pollution simulations are a complex task to perform since the time scales are compared to the spatial scales (micro) for such types of simulation. Boundary and initial conditions for such a simulations are also critical quantities that fundamentally influence the air dispersion results. Microscale Computational Fluid Dynamical Models (CFDM) are playing an increasing role in air quality impact studies for local applications such as new road and building constructions, emergency toxic dispersion gases at urban and local scale, etc. Microscale air dispersion simulations are applied to predict air-flow and pollution dispersion in urban areas, see Pullen et al. (2005). Different combinations and applications appear in the literature such as Pospisil et al. (2004) by integrating a Lagrangian model and a traffic dynamical model into a commercial CFD code, Star-CD to simulate the traffic-induced flow field and turbulence.

In this contribution we have applied the microscale dispersion model MIMO (Ehrhard et al. 2000) to simulate different emission reduction scenarios in Madrid (Spain) related to the vehicle traffic conditions. The MIMO CFD code has been adapted and incorporated into a mesoscale air quality modelling system (MM5-CMAQ-EMIMO) to fit into the one-way nesting structure. MM5 is a meteorological mesoscale model developed by Pennsylvania State University (USA) and NCAR (National Centre for Atmospheric Research, USA) [2]. The CMAQ model is the Community Multiscale Air Quality Modelling System developed by EPA (USA) [1] and EMIMO is the Emission Model developed by San José et al. [4]. MM5 is a well recognized non-hydrostatic mesoscale meteorological model which uses global meteorological data produced by global models such as GFS model (NCEP, USA) to produce high resolution detailed three dimensional fields of wind, temperature and humidity which are used in our case as input for the photochemical dispersion model CMAQ [3]. In addition of MM5 output data, EMIMO model produces for the specific required spatial resolution, hourly emission data for different inorganic pollutants such as particulate matter, sulphur dioxide, nitrogen oxides, carbon monoxide and total volatile organic compounds VOC's. The VOC's are split according to SMOKE (Sparse Matrix Operator Kernel Emissions). (See [5] and [6].)

The CFD and mesoscale models solve the Navier-Stokes equations by using different numerical techniques to obtain fluxes and concentrations at different scales. Mesoscale air quality models cover a wide range of spatial scales from several thousands of kilometres to $1 \mathrm{~km}$ or so. In this contribution we have applied the MM5-CMAQ-EMIMO models over the Madrid region to obtain detailed and accurate results of the pollutant concentrations at this spatial resolution and the MIMO CFD model over a $1 \mathrm{~km} \times 1 \mathrm{~km}$ domain with several spatial resolutions $(2 \mathrm{~m}-10 \mathrm{~m})$ and different vertical resolutions. MM5-CMAQEMIMO data serves as initial and boundary conditions for the MIMO modelling run. 


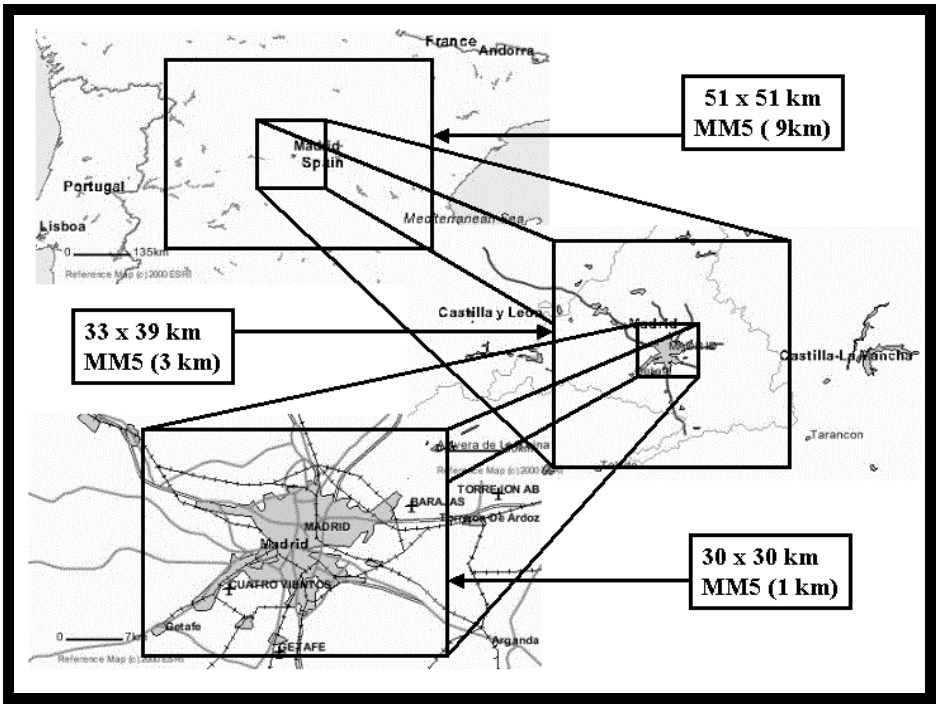

Figure 1: $\quad$ MM5-CMAQ-EMIMO architecture for this application.

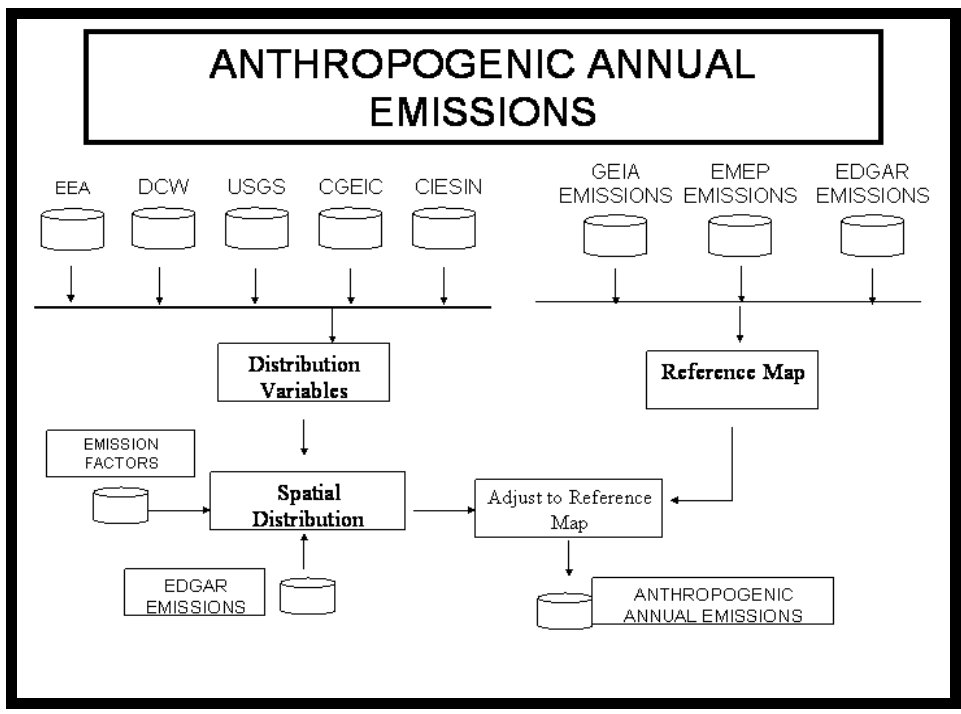

Figure 2: $\quad$ EMIMO model basic architecture.

In Figure 1 we observe the spatial architecture for the application of the MM5-CMAQ-EMIMO mesoscale air quality modelling system. In figure 2 we show a detailed diagram of the EMIMO modelling system. EMIMO is currently operating with the so called Version 2 which includes the CLCL2000 with 44 different landuse types with $100 \mathrm{~m}$ spatial resolution. EMIMO 2.0 also uses the CIESIN 30" [7] global population database and the Digital Chart of the World 
$1 \mathrm{~km}$ land use database to produce adequate emission data per $1 \mathrm{~km}$ grid cell per hour and per pollutant.

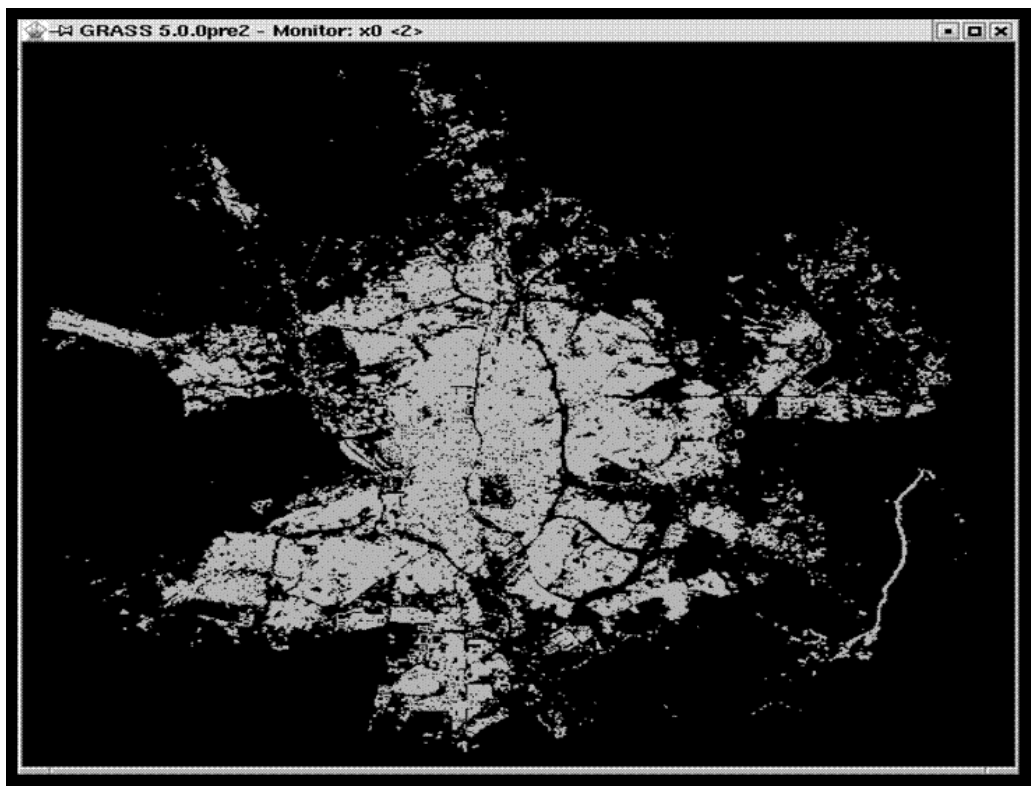

Figure 3: $\quad$ E00 vector file for Madrid Community.

In order to apply the EMIMO CFD model, we need detailed information related to the building structure in the $1 \mathrm{~km}$ grid cell. This information is shown in Figure 3 for the total of the Madrid Community (Spain). The height of the buildings is not included in this file and it has been estimated directly for this experiment. A cellular automata traffic model (CAMO) has been developed. CAMO - which has been included into the EMIMO modelling system-is based on transitional functions defined in a discrete interval $t$ as follows:

$$
\begin{gathered}
p: S x A \rightarrow S \\
v: S \rightarrow U \\
s(t+1)=p(s(t), a(t)) \\
u(t)=v(s(t))
\end{gathered}
$$

where $A$ is a set of input symbols, $S$ is a set of states, $U$ is a set of output symbols, $\mathrm{p}$ and $\mathrm{v}$ are transformations. Transformation $\mathrm{p}$ is a transition function, transformation $\mathrm{v}$ is an output function. Output function $\mathrm{v}$ assigns an output symbol for each state. Transition functions assign change states to next states. Then, $\mathrm{s}(\mathrm{t}+1)$ and $\mathrm{s}(\mathrm{t})$ are defined states at $\mathrm{t}$ and $\mathrm{t}+1$ times, $\mathrm{a}(\mathrm{t})$ is an input symbol, $\mathrm{u}(\mathrm{t})$ is an output symbol. We have used the Moore neighbourhood with 8 
different surrounding cells where each cell - representative of a vehicle - can move on. The whole system focusing on the $1 \mathrm{~km} \times 1 \mathrm{~km}$ urban area in Madrid downtown is called MICROSYS system. We have selected a subdomain of $300 \mathrm{x}$ $300 \mathrm{~m}$ with $5 \mathrm{~m}$ spatial resolution and 15 vertical layers for this particular experiment The first 10 layers are equally spaced with $5 \mathrm{~m}$ spatial resolution up to $50 \mathrm{~m}$ in height and the last five layers are located at $55,61.55,68.20,75.52$ and $83.58 \mathrm{~m}$ in height.

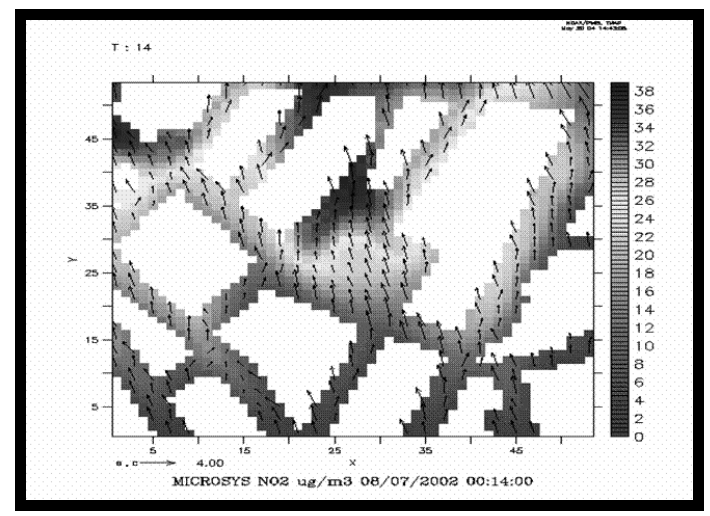

Figure 4: $\quad \mathrm{NO}_{2}$ concentrations at 14:00 July, 8, 2002 produced by MIMO.

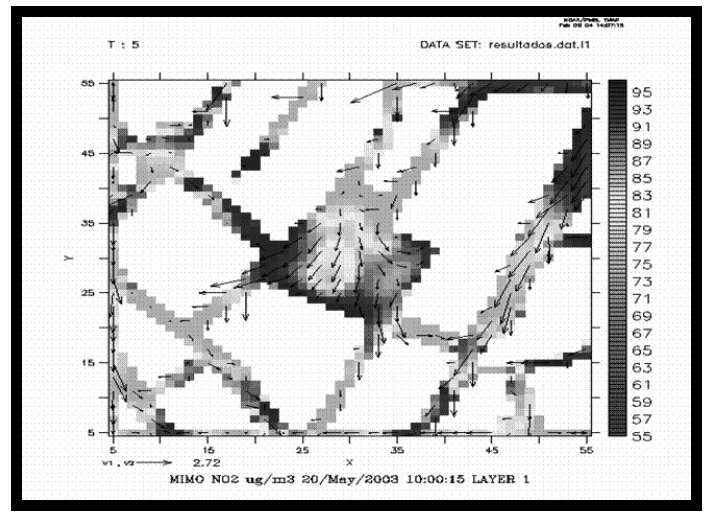

Figure 5: $\mathrm{NO}_{2}$ concentrations at 10:00:15 May, 20, 2003 produced by MIMO.

\section{Results}

We have performed several tests. A preliminary test for July, 8, 2002 at 14:00 is shown in Figure 5 for the $\mathrm{NO}_{2}$ data. In Figure 6 we show the results for May, 20, 2003 at 10:00 hours. A completely different wind pattern is shown in both 
figures. Northern winds are presented early morning on that day and Southern winds are shown at 14:00 on the same day.

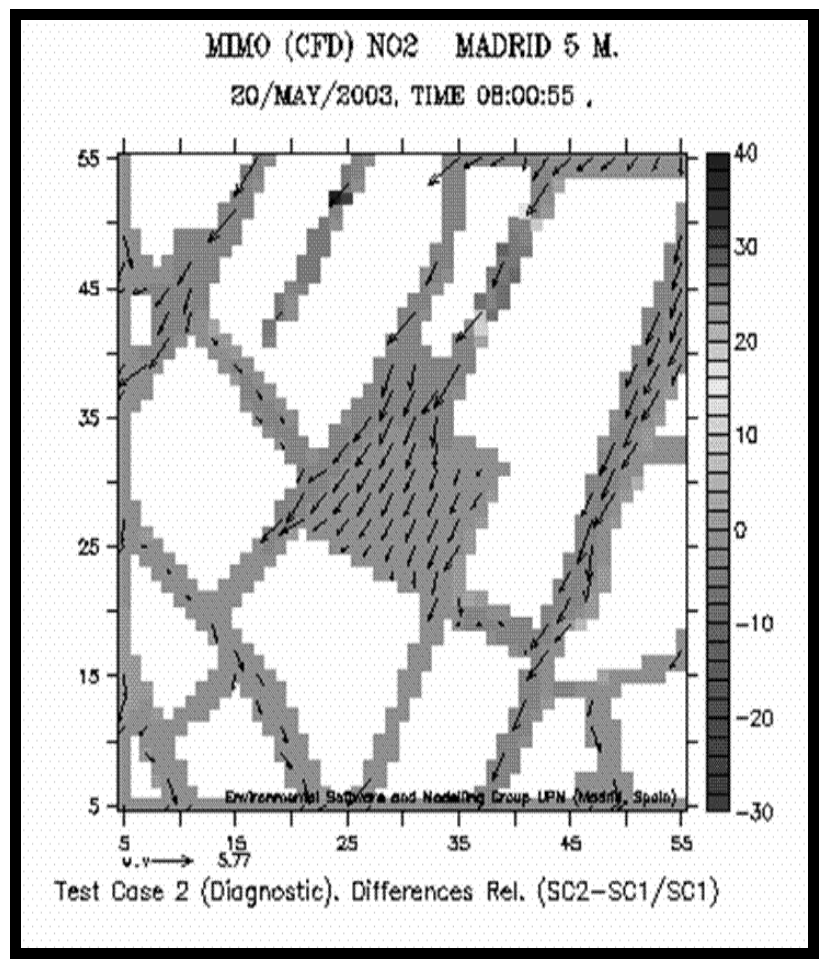

Figure 6: $\quad$ MIMO CFD results for $\mathrm{NO}_{2}$ at 08:00:55 May, 20, 2003 for the differences between scenario b) and scenario a) in percentage respect scenario a). A close-up area is shown in the left lower corner. Differences between $+40 \%$ and $-30 \%$ are found. Most of the highest differences are found in the corners of the streets as expected due to the complexity of the turbulence in those areas.

The complexity of the wind pattern structure is clear from both pictures. In this experiment the prognostic mode has been used for producing both figures 5 and 6. In a further application, the system was applied to produce test results for a synthetic experiment related to the impact of different emission reduction scenarios and shown in the OSCAR EU project (EVK4-CT2002-00083 OSCAR). In this particular application, the system was run for different domains and scenarios in Helsinki (Finland), London (United Kingdom) and Madrid (Spain). In the case of Madrid, two different emission scenarios have been run: a) Normal traffic conditions and b) a decrease of $30 \%$ in the total number of private cars and an increase of $15 \%$ in the total number of public buses. Figure 7 shows the $\mathrm{NO}_{2}$ percentages obtained as differences between the scenario b) and scenario a) divided by the concentrations obtained in scenario a). We observe in 
the lower left corner a detail of a street angle located in the southern area of the square. In the whole domain the impact of a $30 \%$ reduction in the total number of private cars and an increase of $15 \%$ in the total number of public buses has an increase in the air pollution of $40 \%$ in specific areas (normally in the areas located near buildings in the square or in specific "hot spots" in the streets). Most of the values for the "open" areas in the square and streets are on the negative side so that we have a reduction in $\mathrm{NO}_{2}$ concentrations when implementing the emission reduction strategy for this experiment described in scenario b). In Figure 8 we see two details of Figure 7 when we zoom-in into the central and north areas of the domain. We observe that in the square itself most of the data is on the negative side with reductions up to $3.2 \%$ but in the north area we observe that in the street on the left there are two hot spots with the maximum increase and decrease - $+40 \%$ and $-30 \%$ - which gives us a good confidence in model behaviour.

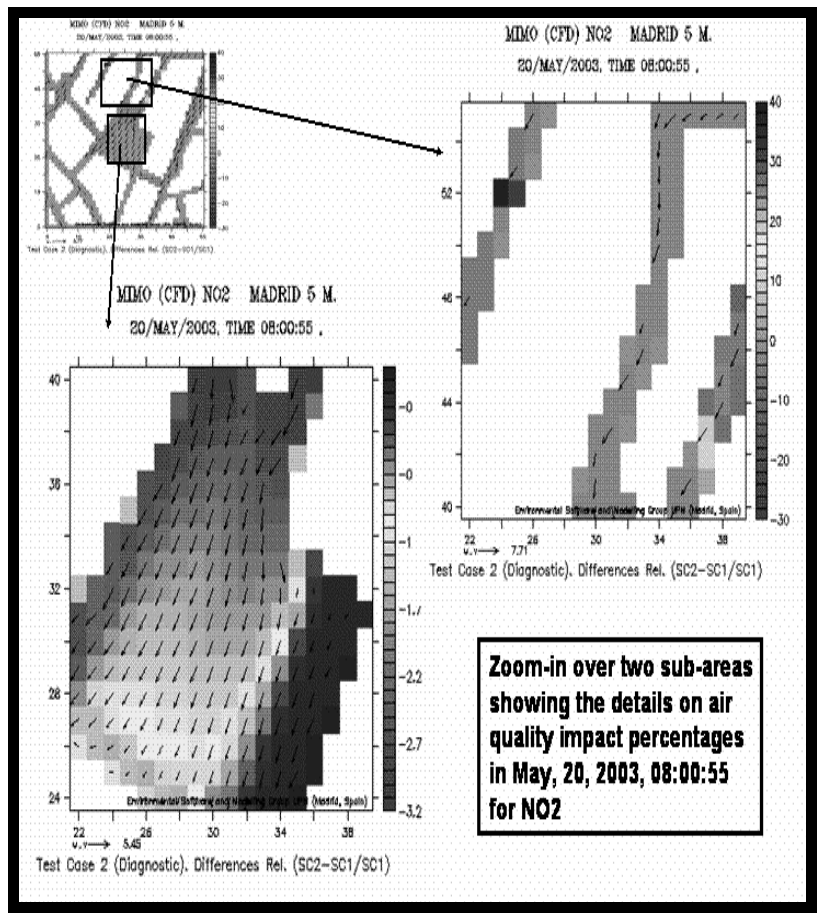

Figure 7: Zoom-in over two sub-areas in model domain for $\mathrm{NO}_{2}$ percentage impact when implementing scenario b) compared with scenario a). The left lower image shows that in square most of the impacts are on the negative side up to $-3.2 \%$ reductions in $\mathrm{NO}_{2}$ concentrations. In the upper-right image, we observe that two grid cells are "hot spots" with the highest increases and decreases in percentage $(+40 \%$ and $-30 \%)$. 
The results show that the integrated system composed by the mesoscale meteorological model MM5, the Community Multiscale Air Quality Modelling System (CMAQ) and the MICROSYS system (composed by adapted MIMO and CAMO models) is producing realistic results although the computer power required is still quite substantial (14 minutes CPU in a PIV-3.4 GHz per 1 minute of simulation in steady state conditions). Further efforts are going to be performed to parallelize the MIMO and CAMO codes to be used in the new generation of dual core processors with an optimum shared memory communication performance.

\section{Conclusions}

The MM5-CMAQ-EMIMO modelling system has been used to provide detailed initial and boundary conditions to a system called MICROSYS which is composed by the MIMO CFD microscale dispersion model and CAMO which is a cellular automata traffic model. The results show that the air quality modelling system offers realistic results although no comparison with eddy-correlation measurement system has been performed in the area. The tool can be used for many air quality impact studies but in particular for traffic emission reduction strategies.

\section{Acknowledgements}

We would like to thank Professor N. Moussiopoulos (AUTH, Greece) for providing the MIMO model. Also to EPA/PSU/NCAR for providing the MM5CMAQ modeling system and OSCAR EU project.

\section{References}

[1] Byun, D.W., J. Young, G. Gipson, J. Godowitch, F. Binkowsky, S. Roselle, B. Benjey, J. Pleim, J.K.S. Ching, J. Novak, C. Coats, T. Odman, A. Hanna, K. Alapaty, R. Mathur, J. McHenry, U. Shankar, S. Fine, A. Xiu, and C. Lang, 1998. Description of the Models-3 Community Multiscale Air Quality (CMAQ) model. Proceedings of the American Meteorological Society $78^{\text {th }}$ Annual Meeting Phoenix, AZ, Jan. 11-16, 1998. pp. 264-268.

[2] Grell, G., J. Dudhia, and D. Stauffer 1994: A Description of the Fifty Generation Penn State/NCAR Mesoscale Model (MM5). NCAR Tech. Note, TN-398+STR, $117 \mathrm{pp}$.

[3] San José R., Prieto J.F., Castellanos N. and Arranz J.M. (1997) Sensititivity study of dry deposition fluxes in ANA air quality model over Madrid mesoscale area, Measurements and Modelling in Environmental Pollution, Ed. San José and Brebbia, pp. 119-130.

[4] San José R., Peña J.I., Pérez J.L. and González R.M. (2003) EMIMO: an emission model. 292-298. ISBN: 3-540-00840-3 Springer-Verlag. 
[5] Williams, A., M. Caughey, H.-C. Huang, X.-Z. Liang, K. Kunkel, Z. Tao, S. Larson, and D. Wuebbles, 2001: Comparison of emissions processing by EM-S95 and SMOKE over the Midwestern U.S. Preprint of International Emission Inventory Conference: One Atmosphere, One Inventory, Many Challenges. Denver, Colorado, May 1-3, pp. 1-13.

[6] Coats, C.J., Jr. 1995. High Performance Algorithms in the Sparse Matrix Operator Kernel Emissions (SMOKE) Modelling System, Microelectronics Center of North Carolina, Environmental Systems Division, Research Triangle Park, NC, 6 pp.

[7] CIESIN, 2004. Center for International Earth Science Information Network (CIESIN). Global Rural-Urban Mapping Project (GRUMP): Urban/Rural population grids. Palisades, NY: CIESIN, Columbia University. http://sedac.ciesin.columbia.edu/gpw/. 